\title{
Number Variance for Arithmetic Hyperbolic Surfaces
}

\author{
W. Luo ${ }^{1}$, P. Sarnak ${ }^{2}$ \\ 1 Mathematics Department, Rutgers University, New Brunswick, NJ 08903, USA \\ 2 Mathematics Department, Princeton University, Princeton, NJ 08544, USA*
}

Received: 28 April 1993

\begin{abstract}
We prove that the number variance for the spectrum of an arithmetic surface is highly nonrigid in part of the universal range. In fact it is close to having a Poisson behavior. This fact was discovered numerically by Schmit, Bogomolny, Georgeot and Giannoni. It has its origin in the high degeneracy of the length spectrum, first observed by Selberg.
\end{abstract}

\section{Introduction}

Let $\lambda_{0} \leq \lambda_{1} \leq \lambda_{2} \ldots$ be a sequence of numbers satisfying

$$
N(x)=\left|\left\{j \mid \lambda_{j} \leq x\right\}\right| \sim x \quad \text { as } \quad x \rightarrow \infty .
$$

There are many statistics that may be used to measure the fine structure of the distribution of the $\lambda^{\prime}$ 's. The one that we will use here is the number variance $\sum^{2}(\lambda, L)$ defined by

$$
\sum^{2}(\lambda, L)=\left\langle(N(\lambda+L)-N(\lambda)-L)^{2}\right\rangle
$$

where \langle\rangle denotes local averaging in $\lambda . \sum^{2}(L)$ measures the variance from the expected number of "levels $\lambda$ " lying in intervals of length $L$. For the local average we choose

$$
\sum^{2}(\lambda, L)=\frac{1}{\lambda} \int_{\lambda}^{2 \lambda}(N(\xi+L)-N(\xi)-L)^{2} d \xi .
$$

Of course, we could replace 2 by $c$, where $c>1$.

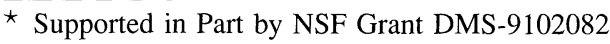

Correspondence to: Dr. P. Sarnak
} 
We also define the average $\bar{\Sigma}^{2}$ by

$$
\bar{\Sigma}^{2}(\lambda, L)=\frac{1}{L} \int_{0}^{L} \sum^{2}(\lambda, u) d u .
$$

The basic models for the distribution of the $\lambda$ 's relevant to spectral problems are

(i) $\lambda_{j}$ 's are random - that is $\lambda_{\jmath+1}-\lambda_{\jmath}$ has a Poisson distribution.

(ii) The $\lambda_{\jmath}$ 's are the eigenvalues of a random symmetric matrix, that is G.O.E. (Gaussian orthogonal ensemble) scaled to satisfy (1.1) [Boh].

(iii) The $\lambda_{j}$ 's are the eigenvalues of a random hermitian matrix, that is G.U.E. (Gaussian unitary ensemble).

For each of these one can compute the expected values of $\sum^{2}(L)$ [B2, Boh]:

(i) Poisson,

$$
\sum^{2}(L)=\sum_{\text {Poisson }}^{2}(L)=L .
$$

(ii) G.O.E. for $L \gg 1$,

$$
\sum^{2}(L)=\sum_{\text {G.O.E. }}^{2}(L)=\frac{2}{\pi^{2}}\left(\log 2 \pi L+\gamma+1-\pi^{2} / 8\right)+O\left(L^{-1}\right) .
$$

(iii) G.U.E. for $L \gg 1$,

$$
\sum^{2}(L)=\sum_{\text {G.U.E. }}^{2}(L)=\frac{1}{\pi^{2}}(\log 2 \pi L+\gamma+1)+O\left(L^{-1}\right) .
$$

Note that the number variance is much smaller for (ii) and (iii) than for (i). We say that the spectrum in (ii) and (iii) is rigid.

Consider now the number variance when the $\lambda$ 's are the eigenvalues of a compact two dimensional Riemannian manifold. In this case after a suitable normalization (1.1) holds this being Weyl's Law. Berry has put forth arguments in favor of the following behavior [B1, B2]:

Fig. 1.

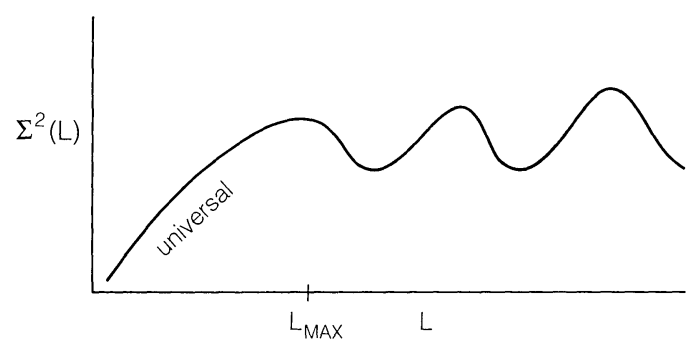

For $1 \ll L \ll L_{\max } \sim \sqrt{\lambda}$ the behavior $\sum^{2}(\lambda, L)$ is universal and given by

(i) $\sum^{2}(L)=\sum_{\text {Poisson }}^{2}(L)$ if the geodesic flow is integrable,

(ii) $\sum^{2}(L)=\sum_{\text {G.O.E. }}^{2}(L)$ if the geodesic flow is chaotic,

(iii) If in case (ii) we break time reversal symmetry by introducing a magnetic field (see below) $\sum^{2}(L)=\sum_{\text {G.U.E. }}^{2}(L)$.

There is a lot of numerical confirmation of these conjectures [B2, BOH]. However a striking violation discovered numerically by $[B-G-G-S]$ and $[A-S]$ concerns the spectrum of an arithmetic hyperbolic surface. 
Let $\Gamma \leq S L_{2}(\mathbb{R})$ be a lattice and $\Gamma \backslash \mathbb{H}$ the corresponding hyperbolic surface (compact). The following characterization of the arithmeticity of $\Gamma$ in terms of the traces of the members of $\Gamma$ is due to Takeuchi [T]. (The reader may use these as definitions.)

Let $\mathscr{L}(\Gamma)=\{\operatorname{trace}(\gamma) \mid \gamma \in \Gamma\}$.

(A) $\Gamma$ is arithmetic iff

(a) $K=Q(\mathscr{L}(\Gamma))$ is a finite extension of $Q$ and $\mathscr{L}(\Gamma) \subset O_{K}$ the ring of integers of $K$.

(b) If $\phi: K \hookrightarrow \mathbb{C}$ is any embedding such that $\left.\phi\right|_{(\mathscr{Y} /(\Gamma))^{2}} \neq$ identity then $\phi(\mathscr{C}(\Gamma))$ is bounded, where $\mathscr{L}(\Gamma)^{2}=\left\{t^{2} \mid t \in \mathscr{L}(\Gamma)\right\}$.

(B) $\Gamma$ is derived from a quaternion algebra iff (a) $\mathscr{L}(\Gamma)$ satisfies $\mathrm{A}($ a) and

(b) If $\phi$ is an embedding of $K$ into $\mathbb{C}$ and $\phi \neq$ id then $\phi(\mathscr{L}(\Gamma))$ is bounded in $\mathbb{C}$.

For any lattice $\Gamma$ it is well known [A-A] that the geodesic flow on $T_{1}^{*}(\Gamma \backslash \mathbb{H})$ is chaotic. Denote by $\lambda_{0}<\lambda_{1} \leq \lambda_{2} \ldots$ the spectrum of the Laplacian $\Delta$ on functions on $\Gamma \backslash \mathbb{H}$ and by $\sum^{2}(\Gamma, \lambda, L)$ the corresponding number variance. In order to break time reversal symmetry and also arithmetic symmetry we consider more generally the spectral problem

$$
\left\{\begin{array}{l}
\Delta \phi+\lambda \phi=0 \\
\phi(\gamma z)=\chi(\gamma) \phi(z), \quad \gamma \in \Gamma, \quad z \in \mathbb{H}
\end{array}\right.
$$

where $\chi: \Gamma \rightarrow S^{1}=\{z|| z \mid=1\}$ is a unitary character of $\Gamma$. This may be thought of as introducing a magnetic field [B-R]. The spectrum for (1.5) is real and will be denoted by $\lambda_{0}(\chi) \leq \lambda_{1}(\chi) \ldots$ The corresponding number variance is denoted by $\sum^{2}(\chi, \Gamma, \lambda, L)$.

The numerical discovery alluded to above is that in the universal range $\sum^{2}(\Gamma, \lambda, L)$ is highly nonrigid for arithmetic $\Gamma$-behaving like $\sum_{\text {Poisson }}^{2}$, while for nonarithmetic

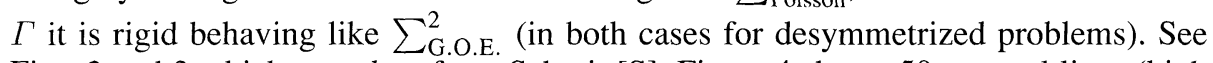
Figs. 2 and 3 which are taken from Schmit [S]. Figure 4 shows 50 spectral lines (high in the spectrum and normalized to have constant mean level spacings) for (a) random numbers (b) the arithmetic group $\Gamma=S L_{2}(\mathbb{Z})$ and (c) the zeros of the Riemann zeta function. Notice that the spectrum of $S L_{2}(\mathbb{Z})$ looks a lot like the random number spectrum while the zeros are rigid, in fact they follow G.U.E. [O]. The Poisson and

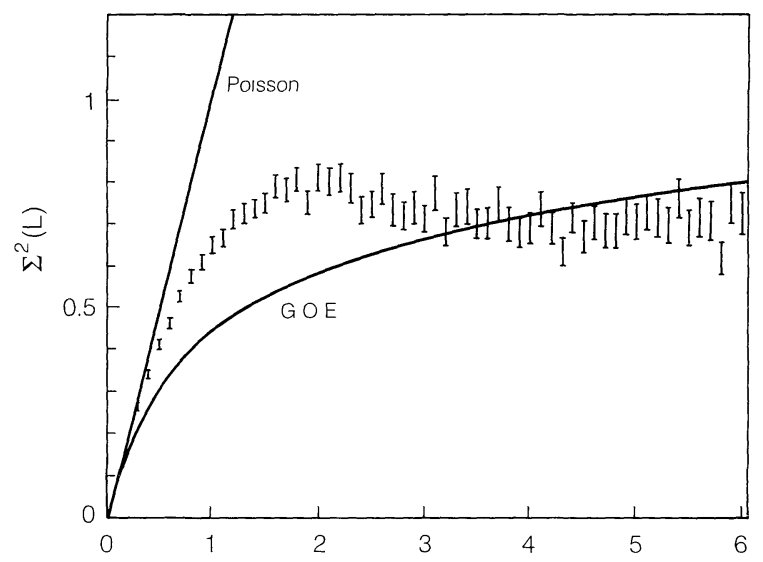

Fig. 2. $\sum^{2}(L)$ for an arithmetic triangle 


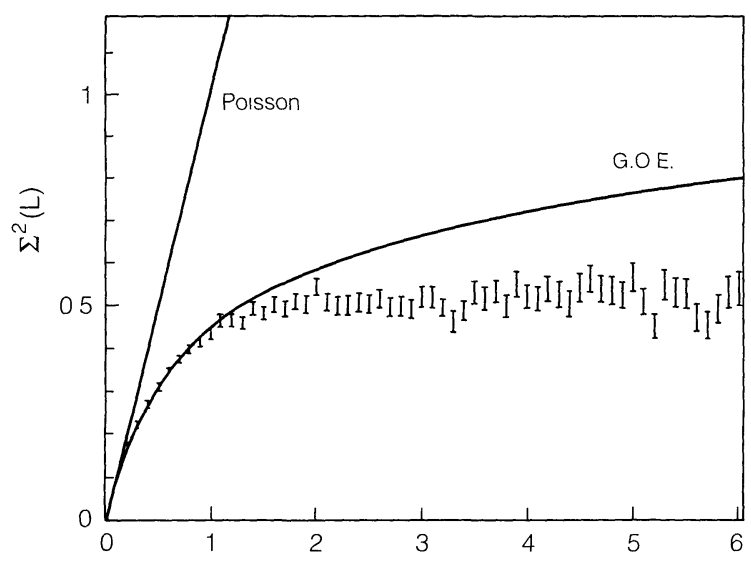

Fig. 3. $\sum^{2}(L)$ for a non-arithmetic triangle

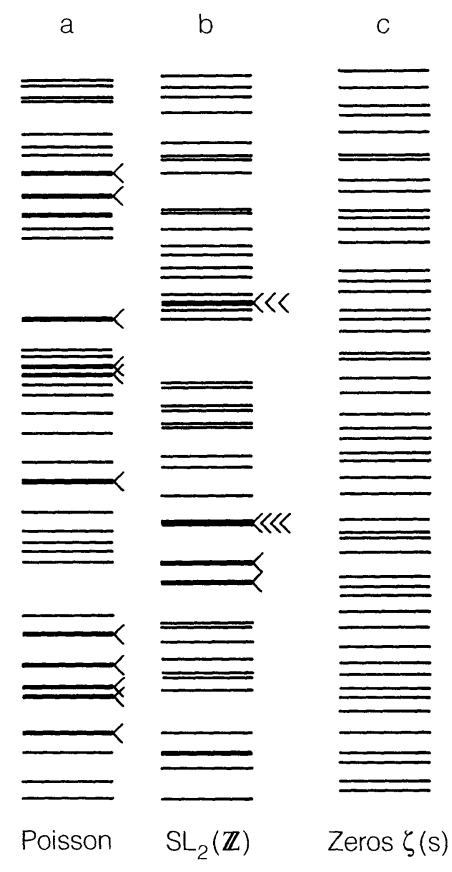

zeros column come from Mehta [M, p. 13]. The $S L_{2}(\mathbb{Z})$ column was drawn using the data in Steil [ST].

Our purpose in this paper is to prove a lower bound on the number variance for arithmetic groups showing that in part of the universal range their spectrum are nonrigid.

Theorem 1.1 If $\Gamma$ is arithmetic then

$$
\bar{\sum}^{2}(\Gamma, \lambda, L) \gg \frac{\sqrt{\lambda}}{(\log \lambda)^{2}}
$$


for

$$
\frac{\sqrt{\lambda}}{\log \lambda} \ll L \ll L_{\max } \cong \sqrt{\lambda} .
$$

Theorem 1.2 If $\Gamma$ is derived from a quaternion algebra then

$$
\bar{\Sigma}^{2}(\chi, \Gamma, \lambda, L) \gg L^{2} \lambda^{2 \delta(\chi)-3 / 2}
$$

for

$$
\lambda^{1 / 4} \ll L \ll \lambda^{1 / 2} / \log \lambda:=\hat{L}
$$

where

$$
\delta(\chi)= \begin{cases}\sqrt{1 / 4-\lambda_{0}(\chi)} & \text { if } \lambda_{0}(\chi) \leq 1 / 4 \\ 0 & \text { otherwise }\end{cases}
$$

Remarks 1.3. (1) For $L \sim \frac{\sqrt{\lambda}}{\log \lambda}$ Theorem 1.1 gives

$$
\bar{\sum}^{2}(\lambda, L) \gg \frac{L}{\log L} .
$$

This is close to establishing and consistent with Poisson behavior in this range.

(2) For $\chi=1, \delta(\chi)=1 / 2$ and Theorem 1.2 gives an effective lower bound for $\sum^{2}$ in the range

$$
\lambda^{1 / 4} \ll L \ll \lambda^{1 / 2} / \log \lambda .
$$

It shows that in this range the spectrum is nonrigid and is Poisson like at least for $L \sim \lambda^{1 / 2} / \log \lambda$.

(3) On introducing $\chi$ the symmetries are broken and so one expects a decrease in $\sum^{2}$ down to G.U.E. However, according to Theorem 1.2, this change cannot happen abruptly (as has been suggested in some other situations [B-R]) because $\lambda_{0}(\chi)$, and hence $\delta(\chi)$ vary continuously in $\chi$. The parameter space for the $\chi$ 's is a torus and the behavior to second order of $\lambda_{0}(\chi)$ for $\chi$ near 1 is determined in [P-S]. The lower bound in Theorem 1.2 gives nonrigid behavior for a range $L \sim \hat{L}$ as long as $\lambda_{0}(\chi)<3 / 16$. It would be very interesting to investigate the rigidity numerically as a function of $\chi$ since it appears that for $\chi$ varying from $\chi=1$ to $\chi=\chi_{0}$ with $\lambda_{0}\left(\chi_{0}\right) \geq 1 / 4$ the statistics may vary continuously from a Poisson to G.U.E. behavior. Moreover throughout the deformation the dynamics are chaotic.

We end the introduction with some comments about the proofs of the theorems. First, the feature of the arithmetic groups that it is at the root of nonrigidity in the spectrum is the high multiplicity in the length spectrum. This was observed in special cases by Selberg and Hejhal $[\mathrm{H}]$ in their derivation of lower bounds for $S(t)$ (see $\left(2.2^{\prime}\right)$ for a definition) and again has been pointed out in [A-S] and [B-G-G-S]. For us the key property of arithmetic groups is the following bounded clustering property (B-C) see Sect. 2.

For $\Gamma$ arithmetic there is $c(\Gamma)<\infty$ s.t.

$$
|\mathscr{L}(\Gamma) \cap[n, n+1]| \leq c(\Gamma) \text { for all } n \in \mathbb{Z} .
$$

If $\Gamma$ is derived from a quaternion algebra then the stronger property of minimal spacing holds:

There is $\delta(\Gamma)>0$ s.t.

$$
\left|\operatorname{trace}(\gamma)-\operatorname{trace}\left(\gamma^{1}\right)\right|>\delta(\Gamma)
$$


if

$$
\text { trace } \gamma \neq \operatorname{trace} \gamma^{1}
$$

In deducing Theorem 1.1 we can only appeal to the weaker B-C property (1.7) and as a result have to face a number of technical problems. To overcome these, we prove some new lower bounds for mean-squares for Dirichlet polynomials (see Lemma 2.3). The reason we only get lower bounds in the theorems is that using the trace formula, one can derive useful expressions for smoothed versions of $N(x)$ - see formula (2.3). A lower bound for the smoothed $\sum^{2}$ gives a lower bound for $\sum^{2}$. Upper bounds for $\sum^{2}$ seem unapproachable by these methods.

\section{Proofs of Theorems}

We begin with proofs of a few useful lemmas, which are of independent interest.

Lemma 2.1. If $\Gamma$ is an arithmetic Fuchsian group, then $\mathscr{L}(\Gamma)$ satisfies the B-C property. Moreover if $\Gamma$ is derived from a quaternion algebra then the stronger property of minimal spacing holds.

Proof. We will use the characterization of arithmetic lattices due to Takeuchi (see Sect. 1). Given $[n, n+1]$, arrange all embeddings of $K=\mathbb{Q}(\mathscr{C}(\Gamma))$ into $\mathbb{C}$ as $\sigma_{1}=\mathrm{id}, \sigma_{2}, \ldots, \sigma_{k}, \sigma_{k+1}, \ldots, \sigma_{n}, n=[K: \mathbb{Q}]$, such that $\sigma_{1}, \ldots, \sigma_{k}$ are all the embeddings satisfying $\left.\sigma\right|_{\mathscr{L}(\Gamma)^{2}}=\mathrm{id}$. Suppose there are more than $2^{k-1}(M+1)$ points of $\mathscr{L}(\Gamma)$ in $[n, n+1], M$ being a positive integer, then by Dirichlet's principle, there are at least two different elements in $\mathscr{L}(\Gamma), \alpha, \beta$, say, such that $0<|\alpha-\beta| \leq \frac{1}{M}$, and $\sigma_{i} \alpha=\varepsilon_{i} \alpha, \sigma_{\imath} \beta=\varepsilon_{\imath} \beta, 1 \leq i \leq k, \varepsilon_{\imath}= \pm 1$. Thus $0 \neq \alpha-\beta \in O_{K}$ and $\left|\sigma_{i}(\alpha-\beta)\right| \leq \frac{1}{M}, 1 \leq i \leq k,\left|\sigma_{i}(\alpha-\beta)\right| \leq 2 A, i>k$. (We assume $\sigma_{\imath}(\mathscr{D}(\Gamma))$ is bounded by $A$ for $i>k$.) Hence $1 \leq\left|N_{\mathbb{Q}}^{k}(\alpha-\beta)\right| \leq \frac{1}{M^{k}}(2 A)^{n-k}$, where $N_{\mathbb{Q}}^{k}$ is the norm from $k$ to $\mathbb{Q}$. When $M$ is sufficiently large the above is impossible. The proof of the second assertion is similar and easier.

Lemma 2.2. Let $a_{j} \geq 0, \lambda_{i} \in \mathbb{R}$. Then there exists $C_{m}>0$, such that for $R \geq 1$,

$$
\int_{R}^{2 R}\left(\frac{1}{2}-\frac{\left|r-\frac{3}{2} R\right|}{R}\right)\left|\sum_{j=1}^{m} a_{j} e^{\imath \lambda} j^{r}\right|^{2} d r \geq C_{m} R \sum_{j=1}^{m} a_{j}^{2} .
$$

Proof. Since $a_{\jmath} \geq 0$, we may assume $\lambda_{j}, 1 \leq j \leq m$ are all different, and satisfy

$$
0=\lambda_{1}<\lambda_{2}<\ldots<\lambda_{m}
$$

moreover we may assume $\sum_{j=1}^{m} a_{j}^{2}=1$.

\footnotetext{
An example of an arithmetic group not satisfying (1.8) is the Hecke group $\Gamma$ generated by $\left[\begin{array}{cc}0 & 1 \\ -1 & 0\end{array}\right],\left[\begin{array}{cc}1 & \sqrt{2} \\ 0 & 1\end{array}\right] \cdot \mathscr{H}(\Gamma)$ contains $2 \mathbb{Z}$ as well as $\sqrt{2} \mathbb{Z}$
} 
We have, after change of variable $r \rightarrow R r$,

$$
\int_{R}^{2 R}\left|\sum_{j=1}^{m} a_{j} e^{i \lambda_{\jmath} r}\right|^{2}\left(\frac{1}{2}-\frac{\left|r-\frac{3}{2} R\right|}{R}\right) d r=R \int_{1}^{2}\left|\sum_{j=1}^{m} a_{\jmath} e^{i R \lambda_{j} r}\right|^{2}\left(\frac{1}{2}-\left|r-\frac{3}{2}\right|\right) d r .
$$

We will conclude the proof if we can prove that

$$
\begin{gathered}
\int_{1}^{2}\left|\sum_{j=1}^{m} a_{j} e^{i \mu_{j} r}\right|^{2}\left(\frac{1}{2}-\left|r-\frac{3}{2}\right|\right) d r \geq C_{m} \quad \text { for } \quad 0=\mu_{1}<\mu_{2}<\ldots<\mu_{m}, \\
\sum_{j=1}^{m} a_{j}^{2}=1, \quad a_{j} \geq 0 .
\end{gathered}
$$

We will use the elementary fact

$$
\int_{R}^{2 R}\left(\frac{1}{2}-\frac{\left|r-\frac{3}{2} R\right|}{R}\right) e^{i r \lambda} d r=O\left(\min \left(R, \frac{1}{\lambda^{2} R}\right)\right)
$$

and proceed by induction.

When $m=1,(2.1)$ is trivial. Suppose (2.1) holds for $m \leq k$, then when $m=k+1$, we have, for $1 \leq v \leq k$,

$$
\begin{aligned}
& \int_{1}^{2}\left|\sum_{j=1}^{k+1} a_{j} e^{\imath \mu_{j} r}\right|^{2}\left(\frac{1}{2}-\left|r-\frac{3}{2}\right|\right) d r \\
& =\int_{1}^{2}\left|\sum_{j=1}^{v} a_{j} e^{i \mu_{j} r}\right|^{2}\left(\frac{1}{2}-\left|r-\frac{3}{2}\right|\right) d r+\int_{1}^{2}\left|\sum_{j=v+1}^{k+1} a_{j} e^{i \mu_{\jmath} r}\right|^{2}\left(\frac{1}{2}-\left|r-\frac{3}{2}\right|\right) d r \\
& \quad+O\left(\sum_{i=1}^{v} \sum_{j=v+1}^{k+1} \frac{a_{\imath} a_{\jmath}}{\left(\mu_{j}-\mu_{i}\right)^{2}}\right) .
\end{aligned}
$$

By induction hypothesis, (2.1) is true unless $\mu_{v+1}-\mu_{v} \leq \Delta(k), 1 \leq v \leq k$, where $\Delta(k)$ is some function of $k$. So we are left with case:

$$
\sum_{j=1}^{k+1} a_{j}^{2}=1, \quad a_{j} \geq 0, \quad 0 \leq \mu_{\imath} \leq k \Delta(k) .
$$

The left-hand side of (2.1) is a positive continuous function in $a_{j}$ and $\mu_{\imath}$, and the above set is compact, hence (2.1) is still true in this case. This completes the proof.

Theorem 2.3. Let $0<t_{1}<t_{2}<\ldots$ satisfy

$$
\left|\left\{t_{\imath}\right\}_{\imath \geq 1} \cap\left[n^{2},(n+1)^{2}\right]\right| \leq C
$$

for all non-negative integers $n ; a_{j} \geq 0$, then there exists $D>0$ such that, for $R \geq 1$,

$$
\int_{R}^{2 R}\left|\sum_{t_{j} \leq N} a_{j} t_{j}^{i r}\right|^{2}\left(\frac{1}{2}-\frac{\left|r-\frac{3}{2} R\right|}{R}\right) d r \geq D R \sum_{t_{j} \leq N} a_{j}^{2}+O\left(\sqrt{N} \sum_{t_{j} \leq N} a_{j}^{2}\right) .
$$


Proof. We can assume $\sqrt{N} \ll R$, since otherwise, Lemma 3 is vacuous.

We define

$$
P_{k}(r)=\sum_{t_{j} \in I_{k}} a_{j} t_{j}^{i r}, \quad k \leq \sqrt{N}
$$

where $I_{k}$ 's are chosen such that $I_{k} \subseteq\left(k^{2},(k+1)^{2}+\delta k\right), I_{k_{1}} \cap I_{k_{2}}=\phi$ if $k_{1} \neq k_{2}$, and $\operatorname{dist}\left(I_{k}, I_{k+1}\right) \geq \delta k$. Here $\delta$ is some positive constant depending on $C$.

Then by Lemma 2.2 and (2.2), we infer that

$$
\begin{aligned}
\int_{R}^{2 R} \mid & \left.\sum_{t_{j} \leq N} a_{j} t_{j}^{\imath r}\right|^{2}\left(\frac{1}{2}-\frac{\left|r-\frac{3}{2} R\right|}{R}\right) d r \\
= & \sum_{k \leq \sqrt{N}} \int_{R}^{2 R} \mid\left(\left.P_{k}(r)\right|^{2}\left(\frac{1}{2}-\frac{\left|r-\frac{3}{2} R\right|}{R}\right) d r\right. \\
& +\sum_{k \neq j} \int_{R}^{2 R} P_{k}(r) \overline{P_{\jmath}(r)}\left(\frac{1}{2}-\frac{\left|r-\frac{3}{2} R\right|}{R}\right) d r \\
\geq & D R \sum_{t_{j} \leq N} a_{j}^{2}+O\left(\sum_{k<\jmath} \sum_{t_{u} \in I_{k}} \sum_{t_{v} \in I_{j}} \frac{a_{u} a_{v}}{R\left(\ln \frac{t_{v}}{t_{u}}\right)^{2}}\right) .
\end{aligned}
$$

We will distinguish two cases.

Case 1. $j-k \geq 2$. The contribution of terms with $j-k \geq 2$ in the last sum

$$
\ll \sum_{j-k \geq 2} \sum_{t_{u} \in I_{k}} \sum_{t_{v} \in I_{j}} a_{u} a_{v} \frac{N}{R(j-k-1)^{2}} \ll \sqrt{N} \sum_{t_{j} \leq N} a_{j}^{2} .
$$

Case 2. $j=k+1$. These terms contribute at most

$$
O\left(\frac{N}{R} \sum_{k \leq \sqrt{N}} \sum_{t_{u} \in I_{k}} a_{u}^{2}\right)=O\left(\sqrt{N} \sum_{t_{\jmath} \leq N} a_{\jmath}^{2}\right) .
$$

This completes the proof.

Lemma 2.4. Let $\delta>0$,

$$
x_{1}<x_{2}<\ldots<x_{R} ; \quad x_{r+1}-x_{r} \geq \delta,
$$

for $1 \leq r \leq R-1$. Then for any complex numbers $b_{r}$, we have

$$
\int_{T}^{2 T}\left|\sum_{r=1}^{R} b_{r} e^{\imath x_{r} t}\right|^{2}\left(\frac{1}{2}-\frac{\left|t-\frac{3}{2} T\right|}{T}\right) d t=\left(\frac{1}{4} T+O\left(\delta^{-1}\right)\right) \sum_{r=1}^{R}\left|b_{r}\right|^{2} .
$$

Proof. The proof is straightforward using (2.2).

Let $\Gamma$ be a cocompact discrete subgroup of $S L(2, \mathbb{R})$, and $\chi: \Gamma \rightarrow \mathbb{C}$ be an unitary character. The hyperbolic Laplacian acts on a dense subspace of the Hilbert space $L_{2}(\Gamma \mid \mathbb{H}, \chi)=\left\{U(z) \mid U(\gamma z)=\chi(\gamma) U(z), \gamma \in \Gamma\right.$, and $\left.\int_{F}|U(z)|^{2} d \mu(z)<\infty\right\}$, 
where $F$ is the fundamental domain for $\Gamma$ and $\mu$ is the invariant measure, and it has discrete spectrum:

$$
0 \leq \lambda_{0}(\chi) \leq \lambda_{1}(\chi) \leq \ldots, \quad \lambda_{n}(\chi) \rightarrow \infty,
$$

where $\lambda_{0}(\chi)=0$ iff $\chi \equiv 1$. Write $\lambda_{j}(\chi)=\lambda_{j}=\frac{1}{4}+t_{j}^{2}=s_{j}\left(1-s_{j}\right), s_{\jmath}=\frac{1}{2}+i t_{j}$, $t_{j} \geq 0$ or $i t_{j} \geq 0$.

Define

$$
N_{1}(T)=\left|\left\{j,\left|t_{j}\right| \leq T\right\}\right|=N\left(\frac{1}{4}+T^{2}\right)
$$

and

$$
S(T)=N_{1}(T)-\frac{\mu(\Gamma \backslash \mathbb{H})}{4 \pi} T^{2}
$$

The prime geodesic theorem $[\mathrm{H}]$ asserts that

$$
\pi_{\Gamma}(x)=\left|\left\{P_{0}, N P_{0} \leq x\right\}\right| \sim \frac{x}{\log x},
$$

as $x \rightarrow+\infty$, where $P_{0}$ runs over the primitive hyperbolic conjugacy classes of $\Gamma$ and $N P_{0}$ the corresponding norm $[\mathrm{H}]$.

If $\left\{P_{0}\right\}$ has trace $l$, then

$$
N P_{0}=x_{l}=\frac{l^{2}}{2}-1+\frac{1}{2} \sqrt{l^{2}-4}=l^{2}-2+0\left(\frac{1}{l^{2}}\right) \text {. }
$$

Define $\mu(l):=\sum_{N P_{0}=x_{l}} \chi\left(P_{0}\right)=\sum_{N P_{0}=x_{l}} \chi\left(P_{0}^{-1}\right)=\overline{\mu(l)}$.

The following lemma shows in particular that $\mu(l)$ is large for arithmetic subgroups and $\chi=1$, a fact first observed by Selberg for quaternion groups (see $[\mathrm{H}]$ ).

Lemma 2.5. Let $\Gamma$ be an arithmetic subgroup of $S L(2, \mathbb{R}), \chi$ is an unitary character of $\Gamma$ such that $\delta(\chi)>0$. Then for any $X \geq 1, b>1$ we have

$$
\sum_{X \leq x_{l} \leq b X} \mu(l)^{2} \gg \frac{X^{\frac{1}{2}+2 \delta(\chi)}}{(\log X)^{2}} .
$$

Proof. Let $u(x)$ be a nonnegative smooth function such that $\operatorname{supp} u(x) \subseteq[1, b]$, and $f(x)=u\left(\frac{x}{X}\right)(\log x)^{-1}$.

Applying the Selberg trace formula $[\mathrm{H}]$ to $g(x)=f\left(e^{x}\right) e^{\frac{x}{2}}+f\left(e^{-x}\right) e^{-\frac{x}{2}}$ and observing $g^{\wedge}(t)=h(t)=\int_{0}^{\infty} f(r) r^{s-1} d r+\int_{0}^{\infty} f(r) r^{\bar{s}-1} d r$ with $s=\frac{1}{2}+i t$, we have

$$
\begin{aligned}
2 \sum_{\{P\}} & \frac{\log N P_{0} \chi(P)}{N P^{\frac{1}{2}}-N P^{-\frac{1}{2}}} f(N P) N P^{\frac{1}{2}} \\
= & \sum_{\jmath} h\left(t_{\jmath}\right)-\frac{\mu(\Gamma \backslash \mathbb{H})}{4 \pi} \int_{-\infty}^{\infty} r \tanh (\pi r) h(r) d r \\
& -\sum_{\{E\}} \sum_{k=1}^{m-1} \frac{\chi^{k}(E)}{m \sin (\pi k / m)} \int_{-\infty}^{+\infty} \frac{e^{-2 \pi k r / m}}{1+e^{-2 \pi r}} h(r) d r .
\end{aligned}
$$


Here $\{P\}$ runs over the hyperbolic conjugacy classes and $\{E\}$ the elliptic classes. Since, by partial integration, $h(t) \ll \frac{X^{\sigma}}{|s|^{p}}$ for any $p>0\left(\sigma=\operatorname{Res}=\operatorname{Re} \frac{1}{2}+i t\right)$ and

$$
2 \sum_{\{P\}} \log N P_{0} f(N P) \chi(P)=\sum_{\frac{1}{2}<\frac{1}{2}+i t_{j} \leq 1} h\left(t_{j}\right)+0\left(X^{\frac{1}{2}}\right) .
$$

Thus, if $\delta(\chi)>0$, then

$$
\begin{aligned}
\frac{X^{\frac{1}{2}+\delta(\chi)}}{\log X} & \ll \sum_{l} \mu(l) u\left(\frac{x_{l}}{X}\right)=\sum_{X \leq x_{l}<b X} \mu(l) u\left(\frac{x_{l}}{X}\right) \\
& \ll\left(\sum_{X \leq x_{l} \leq b X} \mu(l)^{2}\right)^{\frac{1}{2}}\left(\sum_{X \leq x_{l} \leq b X} u\left(\frac{x_{l}}{X}\right)^{2}\right)^{\frac{1}{2}} \\
& \ll b X^{\frac{1}{4}}\left(\sum_{X \leq x_{l} \leq b X} \mu(l)^{2}\right)^{\frac{1}{2}},
\end{aligned}
$$

where we have used Cauchy's inequality and the B-C property which is valid for arithmetic $\Gamma$ by Lemma 2.1. This completes the proof.

The following lemma is related to Proposition 18.11 in Hejhal $[\mathrm{H}]$. It expresses the smoothed mean value of $S(t+u)-S(t), u$ fixed, by a finite Dirichlet polynomial. We have kept our test functions to be quite general and also work directly with the trace formula rather than use the Selberg Zeta function. Note that

$$
N_{1}(T)=\frac{\mu(\Gamma \backslash \mathbb{H})}{4 \pi} \int_{0}^{T} v \tanh \pi v d v+S(T)+0(1)
$$

Let $g(x) \geq 0$ be a smooth even function such that $g(x) \geq 1, x \in\left[-\frac{1}{2}, \frac{1}{2}\right]$ and support $g \subseteq[-1,1]$ and define $h=\hat{g}$, the Fourier transform.

Lemma 2.6. If $10 \leq \beta \leq 2 \log T, 0<u \leq t$ and $t$ is sufficiently large, then

$$
\begin{aligned}
& \int_{|v-t| \leq t / 2}(S(v+u)-S(v)) h(v-t) e^{i \beta(v-t)} d v \\
& =-i \sum_{\substack{l \in \mathscr{L}(\Gamma), e^{\beta-1} \leq x_{l} \leq e^{\beta+1}\\
}} \frac{\mu(l) g\left(\beta-\log x_{l}\right)}{\sqrt{x_{l}}} \\
& \quad \times\left(e^{i t \log x_{l}}-e^{i(t+u) \log x_{l}}\right)+O\left(t^{\varepsilon}\right) .
\end{aligned}
$$

Proof. Consider

$$
I=\int_{0}^{\infty}(S(v+u)-S(v)) h(v-t) e^{i \beta(v-t)} d v .
$$

The trivial bound

$$
S(x)=O(x)
$$


allows us to conclude that

$$
I=\int_{|v-t| \leq t / 2}(S(v+u)-S(v)) h(v-t) e^{i \beta(v-t)} d v+O_{N}\left(t^{-N}\right)
$$

for all $N>0$, since $h$ is rapidly decreasing.

On the other hand

$$
\begin{aligned}
I & =\int_{0}^{\infty} h(v-t) e^{\imath \beta(v-\sigma)}\left(\int_{v}^{v+u} d S(\xi)\right) d v=\int_{0}^{\infty}\left(\int_{\xi-u-t}^{\xi-t} h(y) e^{i \beta y} d y\right) d S(\xi) \\
& =\int_{0}^{\infty} H(\xi) d S(\xi),
\end{aligned}
$$

where

$$
H(\xi)=\int_{\xi-u-t}^{\xi-t} h(y) e^{\imath \beta y} d y
$$

Hence

$$
\begin{aligned}
I & =\int_{0}^{\infty}(H(\xi)+H(-\xi)) d S(\xi)-\int_{0}^{\infty} H(-\xi) d S(\xi) \\
& =\int_{0}^{\infty}(H(\xi)+H(-\xi)) d S(\xi)+O_{N}\left(t^{-N}\right),
\end{aligned}
$$

using again the rapid decrease of $h$.

A calculation yields

$$
\begin{aligned}
& (H(\xi)+H(-\xi))^{\wedge}(x) \\
& \quad=g(\beta-x) e^{-i t x} \frac{1-e^{-i u x}}{i x}-g(\beta+x) e^{i t x} \frac{1-e^{i u x}}{i x} \stackrel{\text { def }}{=} G(x) .
\end{aligned}
$$

Applying Selberg's trace formula to the test function $H(\xi)+H(-\xi)$, we have

$$
\begin{aligned}
\sum_{t_{j}}\left(H\left(t_{\jmath}\right)+H\left(-t_{\jmath}\right)\right)-\frac{\mu(\Gamma \backslash \mathbb{H})}{4 \pi} \int_{-\infty}^{+\infty}(H(r)+H(-r)) \tanh (\pi r) r d r \\
=\sum_{t_{\jmath} \notin \mathbb{R}}\left(H\left(t_{j}\right)+H\left(-t_{j}\right)\right)+\int_{0}^{\infty}(H(r)+H(-r)) d S(r) \\
=\sum_{\{P\}} \frac{\log N P_{0} \chi(P)}{N P^{\frac{1}{2}}-N P^{-\frac{1}{2}}} G(\log N P) \\
\quad+\sum_{\{E\}} \sum_{k=1}^{m-1} \frac{\chi^{k}(E)}{m \sin (\pi k / m)} \int_{-\infty}^{+\infty} \frac{e^{-2 \pi k r / m}}{1+e^{-2 \pi r}}(H(r)+H(-r)) d r
\end{aligned}
$$


Hence, since $H\left( \pm t_{j}\right)=O_{N}\left(e^{\beta / 2} t^{-N}\right), t_{j} \notin \mathbb{R}$, and there are only a finite number of elliptic conjugacy classes in $\Gamma$, we infer that

$$
\begin{aligned}
I= & \operatorname{Re} \sum_{\beta-1 \leq \log N P \leq \beta+1} \frac{\log N P_{0} \chi(P)}{N P^{\frac{1}{2}}-N p^{-\frac{1}{2}}} \\
& \times \frac{e^{-i t \log N_{P}-e^{-i(t+u) \log N_{P}}} g(\beta-\log N P)+O_{N}\left(e^{\beta / 2} t^{-N}\right)}{i \log N P} \\
= & -i \sum_{l \in \mathscr{L}(\Gamma), e^{\beta-1} \leq x_{l} \leq e^{\beta+1}} \frac{\mu(l) g\left(\beta-\log x_{l}\right)}{\sqrt{x_{l}}} \\
& \times\left(e^{-i t \log x_{l}}-e^{i(t+u) \log t_{l}}\right)+O\left(t^{\varepsilon}\right) .
\end{aligned}
$$

This finishes the proof of Lemma 2.6.

We are now ready to prove the main theorems. Let $\beta=2 \ln T-C$, where $C$ is a sufficiently large constant.

Proof of Theorem 1. We have $\chi \equiv 1$.

Define

$$
S_{\beta}(t)=\sum_{l} \eta(l) e^{\imath t \log x_{l}}, \quad T_{\beta}(t)=\sum_{l} \eta(l) e^{\imath(t+u) \log x_{l}}
$$

where

$$
\eta(l)=\frac{v(l)}{\sqrt{x_{l}}}, \quad v(l)=\mu(l) g\left(\beta-\log x_{l}\right) .
$$

$\sum_{l}$ means summation over $l \in \mathscr{L}(\Gamma), e^{\beta-1} \leq x_{l} \leq e^{\beta+1}$. Consider

$$
\begin{aligned}
I= & \int_{T}^{2 T}\left(\frac{1}{2}-\frac{\left|t-\frac{3}{2} T\right|}{T}\right)\left|S_{\beta}(t)-T_{\beta}(t)\right|^{2} d t \\
= & \int_{T}^{2 T}\left(\frac{1}{2}-\frac{\left|t-\frac{3}{2} T\right|}{T}\right)\left|S_{\beta}(t)\right|^{2} d t \\
& +\int_{T}^{2 T}\left(\frac{1}{2}-\frac{\left|t-\frac{3}{2} T\right|}{T}\right)\left|T_{\beta}(t)\right|^{2} d t \\
& -2 \operatorname{Re} \int_{T}^{2 T}\left(\frac{1}{2}-\frac{\left|t-\frac{3}{2} T\right|}{T}\right) S_{\beta}(t) \overline{T_{\beta}(t)} d t \\
= & I_{1}+I_{2}+I_{3}, \quad \text { say. }
\end{aligned}
$$

Evidently $I_{1}, I_{2} \geq 0$.

By Lemmas 2.1-2.3, we infer that

$$
\int_{T}^{2 T}\left(\frac{1}{2}-\frac{\left|t-\frac{3}{2} T\right|}{T}\right)\left|S_{\beta}(t)\right|^{2} d t \gg T \sum_{l} \eta(l)^{2} \stackrel{\text { def }}{=} \Delta .
$$


The same method used in proving Lemma 2.3 leads to

$$
\begin{aligned}
I_{3}= & O\left(\frac{\Delta}{C}\right)-2 \operatorname{Re} \sum_{i \leq \exp ((\beta+1) / 2)} \sum_{\imath^{2}<x_{l_{1}}, x_{l_{2}} \leq(i+1)^{2}} \eta\left(l_{1}\right) \eta\left(l_{2}\right) e^{-\sqrt{-\imath} u \log x_{l}} \\
& \times \int_{T}^{2 T}\left(\frac{1}{2}-\frac{\left|t-\frac{3}{2} T\right|}{T}\right) d t \\
= & O\left(\frac{\Delta}{C}\right)+\tilde{S}, \quad \text { say. }
\end{aligned}
$$

Trivially we have

$$
\int_{U}^{2 U} \tilde{S} d u \ll \frac{\Delta}{\ln T} .
$$

Hence $\int_{U}^{2 U} I d u \gg\left(U-\frac{1}{\ln T}\right) \Delta \gg U \Delta$.

By Lemma 2.6, and Cauchy's inequality,

$$
\int_{U}^{2 U} \int_{T}^{2 T}\left(\int_{|v-t| \leq \frac{t}{2}}|S(v+u)-S(v)| h(v-t) d v\right)^{2} d t d u \gg U \Delta+O\left(U T^{1+2 \varepsilon}\right) .
$$

Since $\int_{-\infty}^{+\infty} h(v) d v=O(1)$, we have, using Cauchy's inequality,

$$
\int_{U}^{2 U} \int_{\frac{1}{2} T}^{3 T}|S(v+u)-S(v)|^{2} d v d u \gg U \Delta+O\left(U T^{1+2 \varepsilon}\right)
$$

Finally,

$$
\Delta \gg \frac{1}{T} \sum_{l} v(l)^{2} \gg \frac{1}{T} \sum_{e^{\beta-\frac{1}{2}} \leq x_{l} \leq e^{\beta+\frac{1}{2}}} \mu(l)^{2} \gg \frac{T^{2}}{(\ln T)^{2}},
$$

by Lemma 2.5 .

Thus, we have proved that

$$
\begin{aligned}
& \int_{U}^{2 U} \int_{T}^{6 T}(S(t+u)-S(t))^{2} d t d u \gg \frac{U T^{2}}{(\ln T)^{2}}, \\
& \quad \text { for } \quad T \geq 4, \quad 1 \gg U \gg \frac{1}{\ln T} .
\end{aligned}
$$

(Here 6 can be replaced by any constant larger than 1 ).

Theorem 1.1 follows from (2.8) using $\left(2.2^{\prime}\right)$ and changing variable. 
Proof of Theorem 2. By Lemma 2.4, (1.8) and (2.2), we infer that

$$
\begin{aligned}
I & \gg T \sum_{l} \eta(l)^{2}\left|1-e^{\imath u \ln x_{l}}\right|^{2} \\
& \gg T \ln ^{2} T u^{2} \sum_{l} \eta(l)^{2} \\
& \gg T^{4 \delta(\chi)} u^{2}, \quad \text { by Lemma } 2.5,
\end{aligned}
$$

provided that $\delta(\chi)>0$ and $u \ll \frac{1}{\ln T}$.

Thus, as before, we obtain

$$
\begin{aligned}
& \int_{U}^{2 U} \int_{T}^{6 T}(S(t+u)-S(t))^{2} d t d u \gg T^{4 \delta(\chi)} U^{3}, \\
& \quad \text { for } T \geq 4,(\ln T)^{-1} \gg U, \text { and } \delta(\chi)>0 .
\end{aligned}
$$

Theorem 1.2 follows from (2.9), (2.3) and a change of variable as before.

Acknowledgement. We would like to thank Ralph Phillips for his comments and careful reading of this paper.

\section{References}

[A-A] Arnold, V.I., Avez, A.: Ergodic problems in classical mechanics. New York: AddisonWesley 1968

[A-S] Aurich, R., Steiner, F.: Energy-level statistics of the Hadamard-Gutzwiller ensemble. Physica D 43, 155-180 (1990)

[A-S-S] Aurich, R., Sieber, M., Steiner, F.: Quantum chaos of the Hadamard-Gutzwiller model. Phys. Rev. Lett. 61, $483-487$ (1988)

[B1] Berry, M.V.: Semiclassical formula for the number variance of the Riemann zeros. Nonlineary 1, 399-407 (1988)

[B2] Berry, M.V.: Some quantum-to-classical asymptotics. Lectures given at the Les Houches School on Chaos and Quantum Physics. Amsterdam: North-Holland 1991, pp. 251-303

[B-R] Berry, M.V., Robnik, M.: Statistics of energy levels without time-reversal symmetry: Aharonov-Bohm chaotic billiards. J. Phys. A., Math. Gen. 19, 649-668 (1986)

[Boh] Bohigas, O.: Random matrix theories and chaotic dynamics. Lectures given at the Les Houches School on Chaos and Quantum Physics. Amsterdam: North-Holland, 1991, pp. 87-199

[B-G-G-S] Bogomolny, E.B., Georgeot, B., Giannoni, M.-J., Schmit, C.: Chaotic billiards generated by arithmetic groups. Phys. Rev. Lett. 69, 1477-1480 (1992)

[H] Hejhal, D.A.: The Selberg trace formula for $P S L(2, \mathbb{R})$. Lecture Notes in Mathematics, Vol. 548. Berlin, Heidelberg, New York: Springer 1976

[M] Mehta, M.L.: Random Matrices. New York: Academic press 1991

[O] Odlyzko, A.: The $10^{20}$ th zero of the Riemann zeta function and 70 million of its neighbors. Preprint

[P-S] Phillips, R., Sarnak, P.: Geodesic in homology classes. Duke Math. J. 55, 287-297 (1987)

[S] Schmit, C.: Quantum and classical properties of some billiards in the hyperbolic plane. Les Houches School on Chaos and Quantum Physics. Amsterdam: North-Holland 1991, pp. 331-369

[St] Steil, G.: Über die Eigenwerte des Laplace Operators und der Hecke Operatoren für $S L(2, \mathbb{Z})$. Thesis, Hamburg

[T] Takeuchi, K.A.: A characterization of arithmetic Fuchsian groups. J. Math. Soc. Japan 27, No. 4, 600-612 (1975)

Communicated by Ya. G. Sinai 